\title{
Energy Model based Performance Analysis of Wireless Sensor Networks
}

\author{
Pooja Jolly \\ Assistant Professor \\ Department of Electronics and \\ Communication Engineering \\ ACME College of Engineering, \\ Ghaziabad, U.P., India
}

\author{
Anjali Goyal \\ Assistant Professor \\ Department of Electronics and \\ Communication Engineering \\ Dehradun Institute of \\ Technology, Dehradun, India
}

\author{
Sandip Vijay \\ Phd, Professor \& HOD \\ Department of Electronics and \\ Communication Engineering \\ Dehradun Institute of \\ Technology, Dehradun, India
}

\begin{abstract}
In this paper, four routing protocols AODV (Adhoc On demand distance vector), ZRP (Zone routing protocol), DSR (Dynamic Source Routing), and DYMO (Dynamic Manet On Demand), are analyzed and compared by using QualNet simulator on the basis of performance metrics such as Throughput, Average End-to-End Delay, Average Jitter, Total Packets Received, Packet Delivery Ratio, Energy Consumption in transmit mode, receive mode, sleep mode and idle mode. The results are taken, examined and analyzed in order to test the efficiency of these four protocols using different energy models. From the study performed it has been analyzed that ZRP consumes maximum energy while other protocols show similar consumption with Generic as the maximum energy consuming mode in transmit receive and idle modes. Mica-motes consumes energy in sleep mode while it shows the least consumption in transmit and receive mode for all the four protocols.
\end{abstract}

\section{General Terms}

Wireless Sensor Networks, QualNet Simulator, Routing Protocols, Energy Model

\section{Keywords}

Wireless sensor network(802.15.4), AODV, ZRP, DSR, DYMO

\section{INTRODUCTION}

A wireless sensor network is composed of a hundreds and thousands of tiny sensors called nodes spread across a geographical area where each sensor functioning autonomously has the capability for wireless communication, signal processing and networking of the data. These nodes will then form a network by communicating with each other. [8]One or more nodes among them will serve as $\operatorname{sink}(\mathrm{s})$ that are capable of communicating with the user either directly or through the existing wired networks. Signals can be transferred without any centralized control and predefined link. Small physical size of these nodes have put a limitation on processing power which further limits the capacity of processor and size of battery. [12] They have a transceiver to communicate with the virtual world and the physical world. Routing topology to be used is decided by the transmission power at nodes and node's location.

There are so many routing protocols available, but in this paper, we are comparing four protocols AODV, ZRP, DSR and DYMO using different energy models(Generic, Micaz, Mica-motes) to analyse Throughput, Average End-to-End Delay, Average Jitter, Total Packets Received, Packet Delivery Ratio, Energy Consumption in transmit mode, receive mode, sleep mode and idle mode. In next sections of this paper, there is a brief discussion of these protocols followed by details of simulation setup and parameters and concluded by simulation results and conclusion.

\section{ROUTING PROTOCOLS}

\subsection{AODV (Ad-Hoc on Demand Distance Vector Routing Protocol)}

The Adhoc On-Demand Distance-Vector Protocol (AODV) [9][10] is a distance vector routing for mobile ad-hoc networks. It is a volatile routing protocol and as the term proposes it ascertains the route on request. [1]AODV shares DSR's on-demand characteristics in that it also discovers route as and when needed by initiating a route discovery process. AODV maintains its routing table with one entry per destination contrary to DSR which preserves multiple route entries for each destination in its route cache. AODV has two phases:- Route Discovery and Route Maintenance. Node that wishes communication with another node has to initiates a Route Discovery Process if it doesn't find any available route to the destination in which a route request packet (RREQ) is broadcasted. Any node which is having a valid route replies with a route-reply by forwarding RREP packet thereby creating a reverse route entry in the routing table. This reverse route entry exists for a span of its lifetime. Hereafter route sighting becomes "on-demand" which reduces the number of prerequisite broadcasts. The "Route Request" packet encompasses the destination node "IP address" viz. the last known sequence number for the destination and hop count. It further comprehends the "Route Request" ID, which is increased every time the nodes initiate a new "Route Request", further the source IP address unruffled with the "Route Request" ID uniquely recognizes a "Route Request" and may be used to distinguish replicas. Furthermore to ensure that the routing statistics are updated, every node preserves a structure number. When the adjoining nodes receive a "Route Request" packet, it paramount generates a "reverse route" to the source node in order to forward responses later. After this it increments the hop count and rebroadcast the "Route Request" packets to its neighbour if it lacks a valid route to the destination .The destination node or the intermediate nodes partaking valid route towards the destination responses with a unicast "Route Request" packet. Thereafter when the "Route Request" is acknowledged, a reverse route towards the originator of "Route Request" is produced. Nodes along the active route broadcast HELLO messages periodically to their neighbours. If the node doesn't obtains a HELLO message or a data packet from the neighbour within a quantified time period, the link between it and the neighbour is consider to be wrecked and is made informed to the source node by sending RERR packet to the neighbours in the "recursion list". The source node after the said link miscarriage may reinitiate the original "discovery process". [9]The second phase of the protocol is the route maintenance. 


\subsection{ZRP (Zone Routing Protocol)}

ZRP is an amalgam variety of routing protocol. [2]Proactive routing uses excess bandwidth to maintain routing information, while reactive routing involves long route request delays. Reactive routing also inefficiently floods the entire network for route determination. The zone routing protocol (ZRP) [2][3] aims to address the problems by combining the best properties of both the proactive and reactive approaches.[3] ZRP is formed by two sub-protocols, a proactive routing protocol: Intra-zone Routing Protocol (IARP), is used inside routing zones and a reactive routing protocol: Inter-zone Routing Protocol (IERP), is used between routing zones. When the route between different zones is required, IERP (Inter zone routing protocol) a reactive protocol used for discovering the route between the source and the destination. This process eradicates the necessity for maintaining the entire picture of the network at every single node. BRP (Border cast resolution protocol) is a technique which controls the traffic between the zones and hence reducing the number furthering in route discovery of IERP. The alterations of the zone radius will further allow the protocol to acclimatize to different WSN environments. Larger radius of the zone will errand proactive routing protocol, which is optimal for slow-moving nodes or large amount of traffic whereas a smaller zone radius will errand the reactive routing protocol, which is best for fast-moving nodes or smaller amount of traffic. ZRP relies on Neighbour Discovery Protocol (NDP) in order to detect the new neighbouring nodes and link failures.

\subsection{DSR (Dynamic source routing protocol)}

DSR[13] is an on-demand routing protocol which is beacon less and hence does not require periodic hello packets. When a source node has a data packet to be sent to the destination, it initiates a Route Request packet which is then flooded throughout the network. On receiving a Route Request each node broadcasts the packet to its neighbours. Each Route Request carries a sequence number generated by the source node and the path it has traversed[14]. Each node performs a sequence number check upon receiving a Route Request packet and forwards it only if it not a duplicate packet. Sequence numbers are used to thwart loop formations and to circumvent multiple transmissions of the same Route Request by an intermediate node, which accepts it through multiple paths. Therefore all the nodes apart from the destination node, forwards a Route Request packet during the route construction phase and a destination node upon receipt of the Route Request packet, responses to the source node through the reverse path the Route Request packet had traversed.

\subsection{DYMO (Dynamic Manet On Demand)}

DYMO uses reactive algorithm and it was being developed in the scope of IETF's MANET working group and considered as a descendant to the AODV routing protocol with the characteristics of path accumulation technique, through which nodes are able to listen to routing messages to acquire information to routes to other nodes without initiating route discoveries themselves. DYMO [7][6] is a routing protocol that was created for situations where clients are mobile and communications is transported through several different clients over a wireless medium, Mobile ad-hoc Network (MANET). Route discovery and route maintenance are the basic operations of DYMO. When the node wishes to discover a path to a destination node, it initiates the route discovery operation and a RREQ message is broadcasted to the network. Every intermediate node contributes in hop-byhop propagation of this message and finds a route to the originator. On reaching the destination node, a RREP packet is send to the originator of the packet through unicast route. A path has now been build up by RREQ which is followed by RREP in backward direction where the route discovered is cached at the source in order to be used for route discovery without sending RREQ.

The route maintenance part of DYMO includes keeping fresh and loop free information with shortest possible path. Link monitoring by using route timers which are updated every time when a packet is forwarded through the route. It also keeps control over route errors if occur by flooding RERR providing information about broken and unreachable nodes.

\section{SIMULATION SETUP}

In this work we have used QualNet 5.0.2 network simulator [11] to evaluate the performance of AODV, ZRP, DSR and DYMO routing protocols of Mobile Ad-Hoc networks and over it CBR traffic is applied and comparison is done using different energy models(Generic, Micaz, Mica-motes).

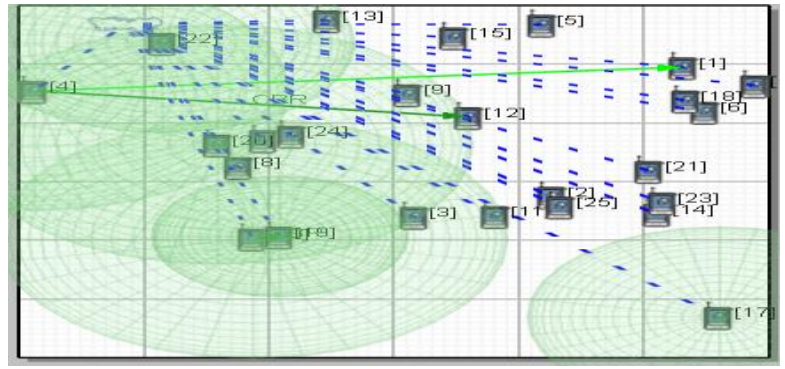

Figure1. Animation View of Wireless Sensor Network (25 nodes)

The simulations are carried out on a network of 25 nodes placed randomly in the area of $600 \mathrm{~m} \times 600 \mathrm{~m}$ where node 1 is a Full Function Device and rest are Reduced Function Devices. CBR applications are applied between source node $(4,19)$ and destination node $(12,10)$ respectively. Simulations are configured for the performance evaluation of different routing protocols with the metrics like Throughput, Average End-to-End Delay, Average Jitter, Total Packets Received, Packet Delivery Ratio, Energy Consumption in transmit mode, receive mode, sleep mode and idle mode.

Table 1. Scenario Parameters

\begin{tabular}{|l|l|}
\hline Parameters & Values \\
\hline Routing protocols & AODV, ZRP, DSR, DYMO \\
\hline Radio type & 802.15 .4 \\
\hline No. of nodes & 25 \\
\hline Area & $600 \mathrm{~m}^{*} 600 \mathrm{~m}$ \\
\hline Data Traffic Type & CBR \\
\hline Mobility of nodes & $\begin{array}{l}\text { Min speed=1m/s, } \\
\text { Max speed=10m/s }\end{array}$ \\
\hline Mobility & Random Way Point \\
\hline Path loss model & Two Ray \\
\hline Energy model & 1. Generic, 2.Mica- motes, 3.Micaz \\
\hline Simulation time & 600 seconds \\
\hline
\end{tabular}




\section{RESULTS AND DISCUSSIONS}

\subsection{Throughput}

The phrase "Throughput" can be elucidated as the average rate of successful data packets received at its destination where it may be transported over a physical or logical linkage or passed over a certain network node. Throughput is customarily measured in bits per second (bit/s or bps) and occasionally in data packets per second.

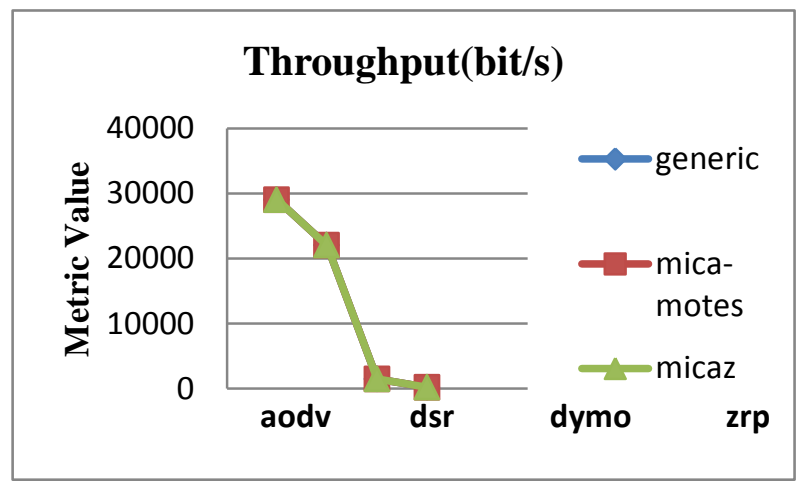

Figure2. (a) Graph of Server throughput

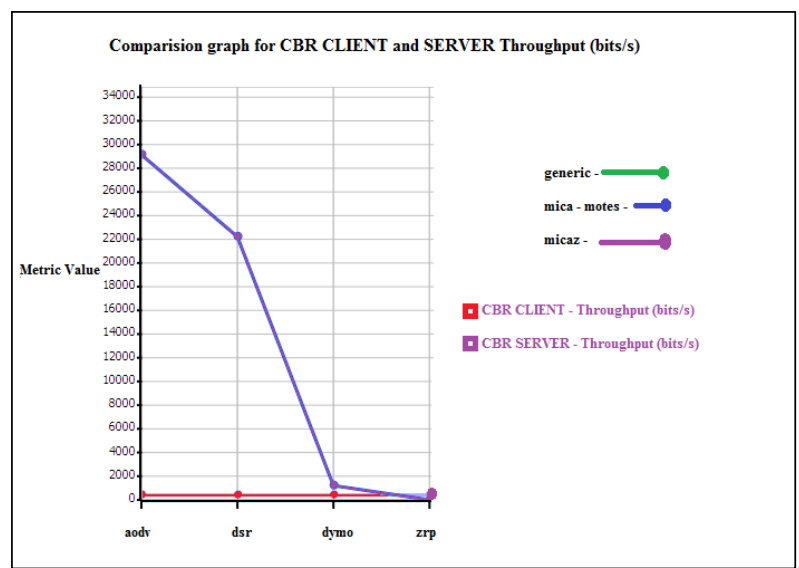

Figure2. (b) Comparison graph of server and client throughput

Figure2 depicts the variation of throughput for four routing protocols reflected for different energy models. From figure 2 it has been scrutinized that throughput is maximum for AODV which is tailed by DSR then DYMO and minimum for ZRP. ZRP gives the minimum throughput for all three energy models.

\subsection{Average End To End Delay}

It is the time taken by a packet to reach from source to destination. The average end-to-end delays over all the received packets is known as the average end-to-end delay. The delay metric of a packet includes delays due to route discovery, queuing, propagation time and transfer time.

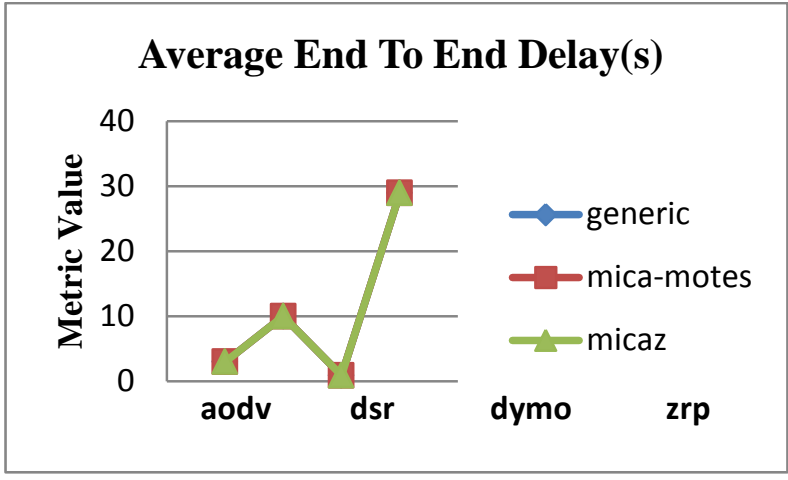

Figure3. Graph for End To End Delay

Figure 3 shows the variation of end to end delay for various routing protocols considered for different energy models. From figure 3 it has been analysed that average end to end delay is least for DYMO followed by AODV, DSR and maximum for ZRP. ZRP gives the highest average end to end delay for all the three energy models.

\subsection{Average Jitter}

The variation in the latency of packets at the destination is termed as Jitter which is caused due to congestion, topology change etc. in a network. Its value is required to be as low as possible for the better performance of any protocol.

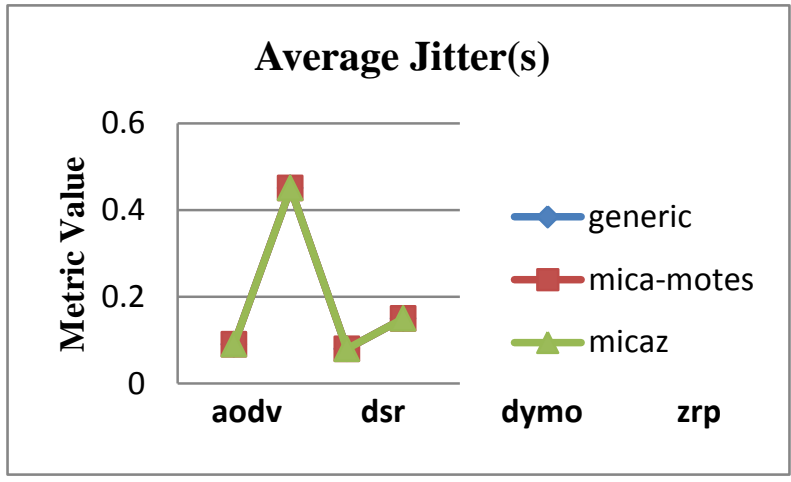

Figure4. Graph for Average Jitter

Figure4 shows the variation of average jitter for various routing protocols considered for different energy models. From figure 4 it has been analysed that average jitter is least for DYMO followed by AODV then ZRP and then DSR. DSR gives the maximum value of average jitter for all the three energy models.

\subsection{Total Packets Received}

Total packet received by any server per second determines the efficiency of the network for delivering the packet without loss. More is the number of packets received per unit time more will be the efficiency of the network. 


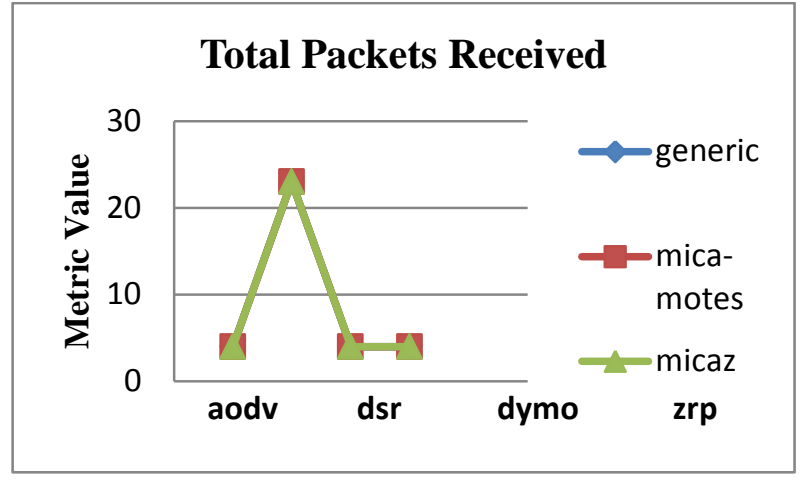

Figure5. Graph for Total Packets Received

On analyzing the above graph it is concluded that using DSR there are maximum number of packets received while packets received are same in other three protocols in all the three energy models.

\subsection{Packet Delivery Ratio}

It is the measure of the ratio of the number of data packets received by the destination to the total number of packets generated at the source. Its higher value shows the quality and correctness of a protocol.

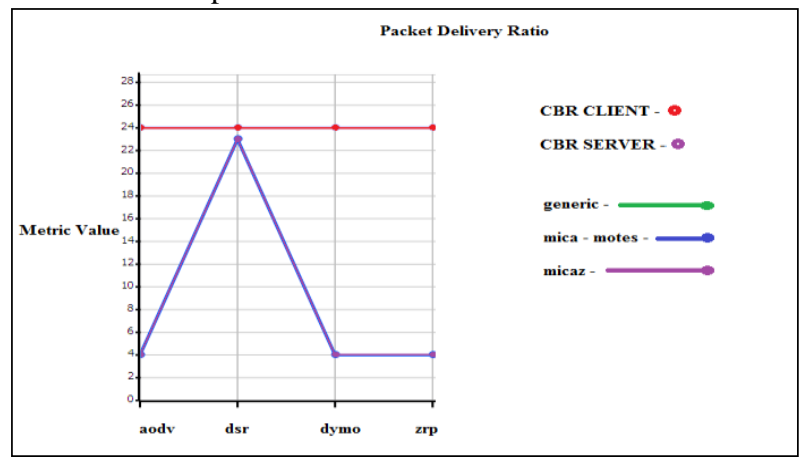

Figure6. Packet Delivery Ratio

On analyzing the above graph it is depicted that maximum number of packets has been sent in DSR in all the three energy models(Generic, Mica-Motes, Micaz)while packet delivery is same in AODV, DYMO and ZRP.

\subsection{Energy Consumption}

The lifetime, scalability, response time and effective sampling frequency, all these parameters of the WSN depend upon the power. Power failure often because breakage in network. Energy is required for maintaining the individual health of the nodes, during receiving the packets and transmitting the data as well.

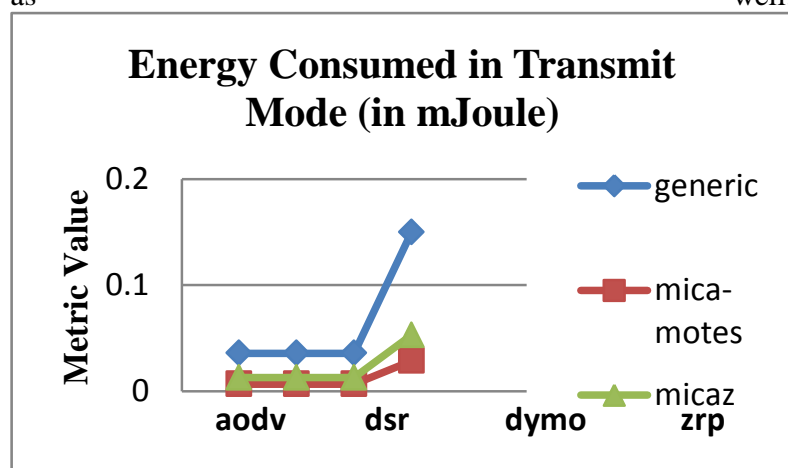

Figure6. (a)Graph for energy consumed in transmit mode

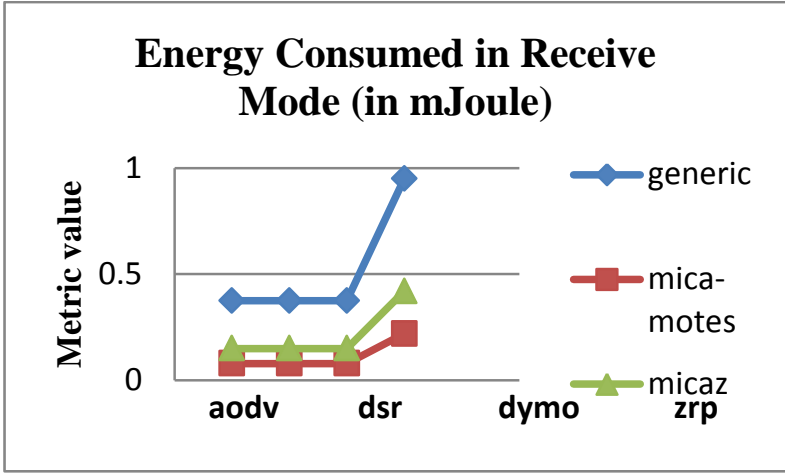

Figure6. (b)Graph for energy consumed in receive mode

On analyzing the results for energy consumption in transmit and receive mode it has been concluded that ZRP consumes maximum energy while energy consumption for the rest three protocols is same in all the three energy models. Energy consumption is minimum in Mica-motes followed by Micaz and maximum in Generic for both transmit and receive modes.

\section{Energy Consumed in Sleep Mode (in mJoule)}

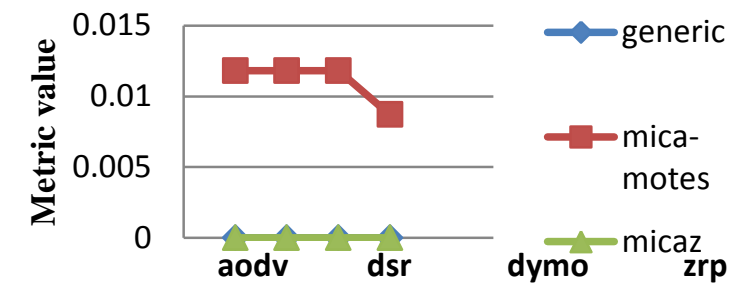

Figure6. (c) Graph for energy consumed in sleep mode

From the above graph it is concluded that there is a considerable consumption of energy in sleep mode when Mica-motes is used while energy consumption is negligible with Generic and Micaz. Among the four protocols ZRP consumes minimum energy while other three protocols consume similar energy in Mica-motes during sleep mode.

\section{Energy Consumed in Idle Mode}

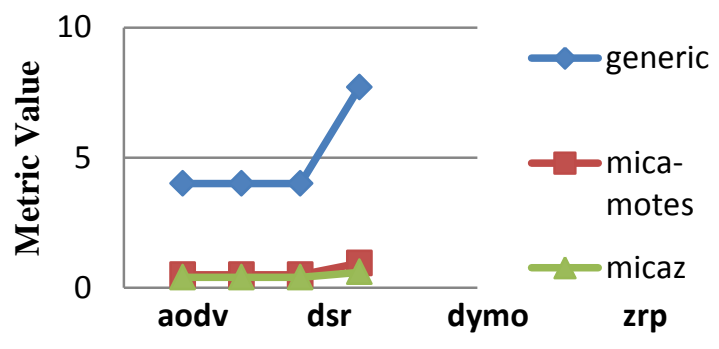

Figure6. (d)Graph for energy consumed in idle mode

On analyzing the results for energy consumption in idle mode it is noted that there is minimum consumption in Micaz followed by Mica-motes and maximum in Generic. Among the routing protocols ZRP shows the maximum consumption in all the three energy models while consumption is same for the other three protocols. 


\section{CONCLUSIONS}

From the above data obtained it has been analyzed that throughput is maximum for AODV followed by DSR, DYMO and minimum for ZRP. ZRP gives the minimum throughput for all three energy models. Average jitter is least for DYMO followed by AODV then ZRP and then DSR. DSR gives the highest Average jitter value. Average end to end delay is least for DYMO followed by AODV, DSR and ZRP and thus ZRP gives the highest average end to end delay. Maximum number of packets has been sent in DSR in all the three energy models while packet delivery is same in AODV, DYMO and ZRP. For energy consumption in transmit, receive mode and idle mode it has been concluded that ZRP consumes maximum energy while other protocols show similar consumption with Generic as the maximum energy consuming mode. Micamotes has been proved the only energy consuming model in sleep mode with ZRP shows the minimum consumption.

From the study performed it has been analyzed that only Mica-motes consumes energy in sleep mode while it shows the least consumption in transmit and receive mode for all the four protocols.

\section{REFERENCES}

[1] N.Vetrivelan and A.V.Reddy, "Performance Analysis of Three Routing Protocols for Varying MANET Size", Proceedings of the International Multi-Conference of Engineers and Computer Scientists 2008, Vol. II (ISBN:97988-17012-1-3).

[2] Subramanya Bhat.M, Shwetha.D and Devaraju.J.T, "A Performance Study of Proactive, Reactive and Hybrid Routing Protocols using Qualnet Simulator" International Journal of Computer Applications (0975 - 8887), Vol. 28-No.5, August 2011, pp-117.

[3] SreeRanga Raju and Jitendranath Mungara, "Performance Evaluation of ZRP over AODV and DSR in Mobile Adhoc Networks Using Qualnet" European Journal of Scientific Research, Vol. 45, No.4. 658-674, 2010.

[4] Wireless routing protocols www.wikipedia.com.

[5] QualNet Network Simulator. www.scalablenetworks.com.

[6] Chakeres, CenGen, WiChorus, etc., "Dynamic MANET On-demand(DYMO)Routing[Z]'Intemet-Draft,draft-ietfmanet-dymo-21(work in progress), July 26, 2010.
[7] Archie Budhiraja and Roopali Garg, "Performance Comparison of Dynamic Mobile ad-hoc network ondemand multipath routing protocol with AODV" International Journal of Scientific \& Engineering Research ( ISSN 2229-5518), Volume 2, Issue 11, November-2011, pp1-9.

[8] Rajashree.V.Biradar, V.C.Patil, Dr. S.R.Sawant and Dr.R.R.Mudholkar, "CLASSIFICATION AND COMPARISON OF ROUTING PROTOCOLS IN WIRELESS SENSOR NETWORKS", Special Issue on Ubiquitous Computing Security Systems, UbiCC Journal, Vol.4, pp704-711.

[9] Parma Nand and Dr. S.C. Sharma, "Performance study of Broadcast based Mobile Adhoc Routing Protocols AODV, DSR and DYMO", International Journal of Security and Its Applications, Vol.5 No. 1, January, 2011, pp53-64.

[10] Charles Perkins, Elizabeth Royer, and Samir Das, "Ad hoc on demand distance vector (AODV) routing", IETF RFC No. 3561, July 2003.

[11] QualNet documentation, "QualNet 5.0.2 Model Library:AdvancedWireless".

[12] D.D.Chaudhary, Pranav Panwar and Dr.L.M. Waghmare, "Comparison and Performance Evaluation of Wireless Sensor Network with different Routing Protocols", International Conference on Information and Electronics Engineering, IPCSIT Vol.6, 2011, pp-278-282.

[13 ]E.M.Royer and C.K.Toh, "A Review of current Routing Protocols for Ad-Hoc Mobile Wireless Networks", IEEE Personal Communications Magazine, 1999, pp-46-55.

[14] Vivek Thaper, Bindyia Jain and Varsha Sahni, "PERFORMANCE ANALYSIS OF ADHOC ROUTING PROTOCOLS USING RANDOM WAYPOINT MOBILITY MODEL IN WIRELESS SENSOR NETWORKS", Vivek Thaper et al. / International Journal on Computer Science and Engineering (IJCSE), Vol. 3 No. 8 (ISSN : 0975-3397), August 2011, pp3059-3060. 